\title{
Effect of metal foam on vibration damping and its modelling
}

\author{
Paolo Albertelli ${ }^{1,2}$ (i) $\cdot$ Stefano Esposito ${ }^{1} \cdot$ Valerio Mussi $^{2} \cdot$ Massimo Goletti $^{2} \cdot$ Michele Monno $^{1,2}$
}

Received: 20 December 2020 / Accepted: 28 April 2021 / Published online: 18 May 2021

(C) The Author(s) 2021

\begin{abstract}
The use of metal foams for damping vibrations of mechanical structures has found interesting applications in machine tools and its components. Indeed, undesired vibration is one of the most detrimental causes that limit the machine performance in terms of the maximum achievable material removal rate MRR. Although positive results were presented in some research works, a methodology for predicting the damping properties of such materials in the machine tool design phase using finite element codes is still missing. In order to bridge this gap, in this paper, an experimental procedure for identifying the damping contribution of the aluminum metal foam to the hosting structure is proposed. The experimental data are even used to develop a model for predicting the damping. The procedure is further validated on a dummy structure.
\end{abstract}

Keywords Metal foam · Damping modelling

\section{Introduction}

The performance of machine tools can be affected by the presence of undesired vibrations, which negatively influence the maximum achievable material removal rate (MMR) and the tracking accuracy of axis. Furthermore, vibrations can lead to the failure of components such as spindle bearings. This issue becomes more relevant in high-speed machining, in which the high cutting speeds lead to major problems concerning the dynamic of machine tools and vibrations of its components.

Therefore, dynamic characteristics of mechanical structures become fundamental in order to avoid dangerous vibrations. In this scenario, damping properties, which are the capacity of reducing oscillations by dissipation of mechanical energy, assume a key role. Material (or internal) damping and structural damping are the main source of damping to be

Peer-review statement: Peer review is under the responsibility of the scientific committee of the International Conference on Advanced and Competitive Manufacturing Technologies.

Paolo Albertelli

paolo.albertelli@polimi.it

1 Mechanical Engineering Department, Politecnico di Milano, Via La Masa 1, 20156 Milan, Italy

2 Consorzio MUSP, Strada Torre della Razza, 29122 Piacenza, Italy considered in the field of machine tools, and they originate from different physical phenomena. Indeed, material damping is caused by microscopic and macroscopic dissipating processes within the material, while structural one depends on relative motions between components in correspondence of points of contact, joints, or supports [1].

The dynamic performance of machine tools can be increased using innovative materials, such as metal foams. Indeed, this family of materials has a high internal damping capacity, which is typically five to ten times greater than that of the metal from which it is made [2]. Furthermore, the use of metal foams as a filler (e.g., as foam sandwiches or foam-filled tubes) allows to increase the structural damping properties of the machine. Therefore, metal foams allow to minimize vibrations, increase the MMR, and guarantee a good workpiece quality, but estimation and prediction of damping in mechanical structures, filled by these materials, still remain challenging. Indeed, the prediction of damping of complex structures can be extremely useful tool during the design phase, which allows to reduce the need of creating expensive prototypes, too.

So far, many research studies were performed about the damping properties of metals (metal foams included). More specifically, experimental procedures to identify damping parameters such as loss factor or damping ratio were conceived. These works were typically supported by FEM models, which were used to validate the experimental tests or as preliminary tools (for the setup of test parameters). Formulations for predicting the damping properties of foamed structures, to 
be used in combination with FEM models during the design phase of the machine tools, are still missing.

Goletti et al. [3] proposed an industrial-level experimental procedure to estimate material damping of metal foams, in terms of damping ratio parameter. Then, they replicated the test by a FEM model, in which the experimental damping ratio values were used. The procedure was validated. The main limit of this method is that it does not consider the damping behavior of the contact between foam and skin, i.e., the structural damping.

Arora [4] developed a method to identify structural damping matrix of simple metallic components, such as cantilever beams from complex FRFs (frequency response functions) that were experimentally obtained. This method was then validated by a representative FEM model. It showed good agreement between experimental and numerical FRFs, but its usage was limited to simple structures, and it is strongly dependent on the considered range of frequency.

Aggogeri et al. [5] compared dynamic performance of a test ram structure, made of different hybrid materials, aluminum foam sandwiches included. First, they performed a modal analysis by FEM models to analyze the main vibration modes. Then, they executed an experimental modal analysis to compute FRFs of the prototypes and estimate the structural damping factor. Their work confirmed the high damping property of innovative materials like the metal foams.

Strano et al. [6] carried out some experimental tests on foamed tubes considering different interface conditions, in order to understand their influence on structural damping. They used a FEM model, representative of the real test bench, in order to have preliminary information about the modes of the analyzed structure. Finally, they conducted an experimental modal analysis to estimate the damping ratio of the tubes. With this research, it was demonstrated that interfaces strongly influence the structural damping value.

In the abovementioned research works, different experimental procedures, supported by FEM simulations to estimate damping in mechanical structures, are presented. The main limitation of these methods is that the damping values, so estimated, are valid only for the specific evaluated structures, and they do not allow to predict the damping behavior of new systems, which are different from the tested ones.

In this paper, a model to predict damping properties of metal foams in complex structures was presented. In the first part of this work, experimental tests and FEM simulations are performed on simple structures, in this case on aluminum foam filled tubes, in order to define critical damping values, which guarantee a good agreement between experimental and simulated FRFs. Based on the obtained results, a predictive model of damping factors was developed.

In the second part, the predictive model is validated on the basis of two different structures. The first one is again an aluminum foam filled tube, but with different dimensions if compared to the previous ones, and the second one is definitely a more complex structure, which is composed of various foamed tubes. In both cases, the model is used to predict damping coefficients, which are then applied in the FEM simulations, in order to obtain the FRF of the system. Finally, the accuracy and the reliability of the model formulation were confirmed by the comparison of simulated FRF and experimental ones.

\section{Materials and methods}

In the following sections, the experimental tests performed on foamed filled tubes, the corresponding FEM models and the novel damping predictive model, developed during this research project, are presented.

\subsection{Experimental tests}

Experimental tests are carried out on aluminum foam filled tubes, in order to obtain their FRFs. Two different configurations were evaluated during the experimental phase (see Table 1).

An instrumented hammer (PCB model 086E80) is used to excite the free end of the tube, and an accelerometer (PCB model $352 \mathrm{C} 23$ ) is placed opposite to the driving point, in order to acquire the FRF for both the configurations (see Fig. 1). The other tip of the tube was connected to a massive concrete block by means of a circular support and a smaller flange.

Both the components were made of steel and connected by bolts (see Figs. 1 and 2). Several types of connection (welding, glue, etc.), between the tube and the flange, were tested to minimize the damping effect of the connection interface and to evaluate the damping contribution due to the tubes only (see Fig. 3). In addition, the contact area is reduced by means of a step profile (see Fig. 4). Steel washers are used at the interface between flange and circular plate to reduce contact area between these components too.

For sake of simplicity, this part of the test was not presented in the current paper. In the next paragraphs, all the results are referred to the case with the welded stepped tube, for which

Table 1 Configuration of tested tubes

\begin{tabular}{lll}
\hline Parameter & Conf. A & Conf. B \\
\hline Tube length (mm) & 190 & 190 \\
Tube outer diameter (mm) & 8 & 12 \\
Tube thickness (mm) & 1 & 1.5 \\
Tube material (/) & Steel (S235) & Steel (S235) \\
\hline
\end{tabular}




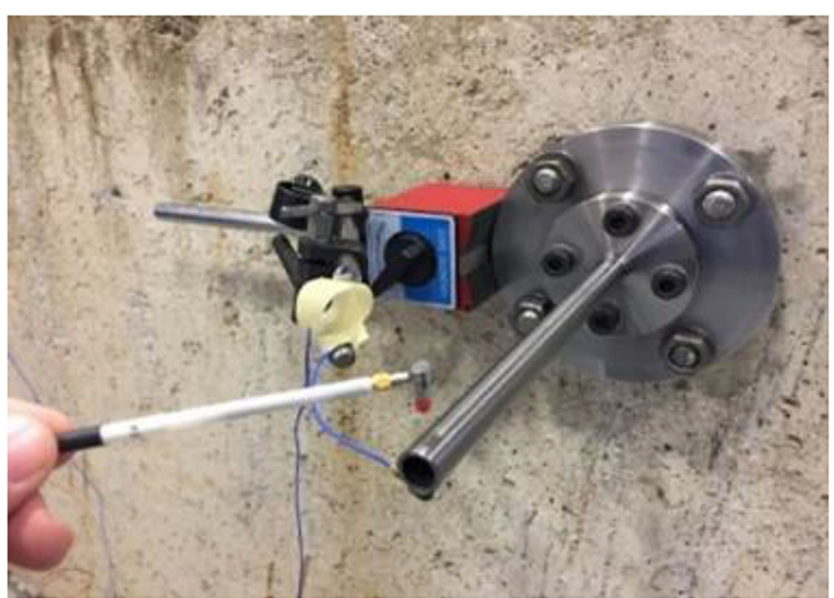

Fig. 1 Experimental setup

the damping contribution of the connection interface can be neglected.

Preliminary tap tests on empty tubes were performed for both the configurations, in order to establish the structural damping value of the steel tubes $(0.001)$, which has been used for all the FEM models. In most experimental cases, the half power bandwidth technique is used to identify damping coefficients. This technique belongs to the category of single degree-of-freedom (SDOF) methods, which are based on the one degree of freedom system, modeled by mass-springdamper system, but half power bandwidth method may be affected by not negligible errors, due to poor frequency resolution (see [3]). In the current research work, in order to avoid the abovementioned estimation errors, the circle fit method is used for the damping estimation [7]. It is a SDOF method, based on a regression in the Nyquist plane. It is more accurate than the half power bandwidth method since it considers more points for the modal parameter estimation. It is a SISO technique (i.e., a single input is applied and a single response is measured), which is applied in a recursive way. This procedure can be used since the distance in frequency domain between eigenmodes is high.

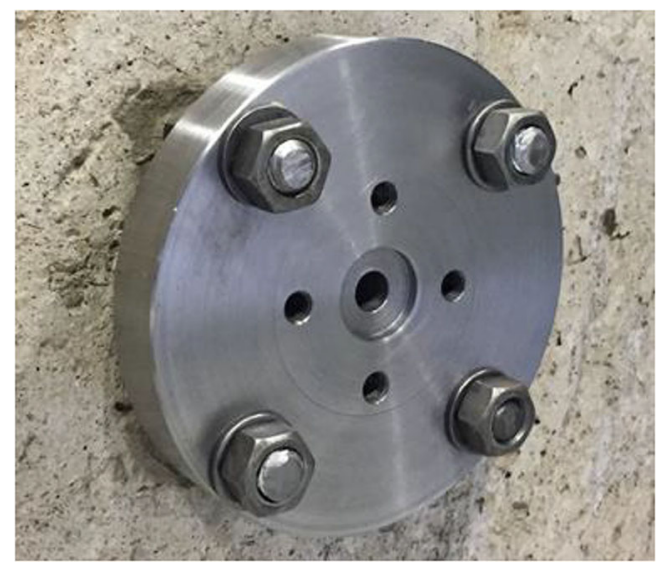

Fig. 2 Circular support

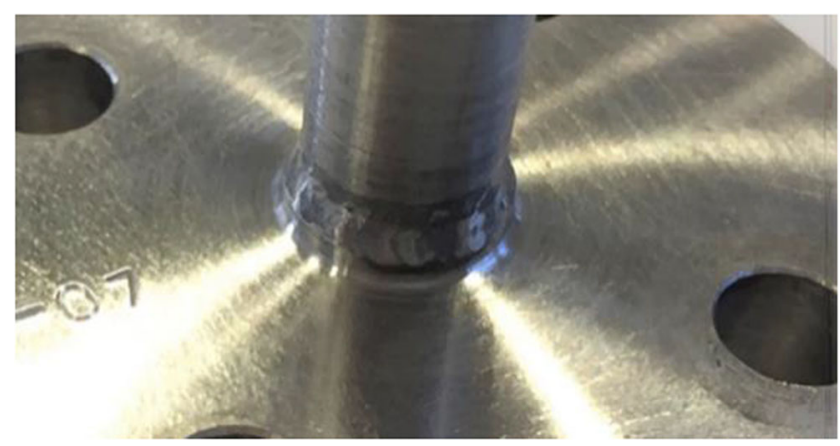

Fig. 3 Welded tube

In the following tables, the preliminary (Table 2) and main test cases have been summarized.

As shown in Table 3, the material used for the foam production process was Alulight, which has been obtained by direct extrusion of a $\mathrm{AlSi} 10+0.8 \% \mathrm{wtTiH}_{2}$ powder (i.e., $90 \%$ aluminum, $10 \%$ silicon, and $0.8 \%$ by weight of titanium hydride). The experimental results are presented in Tables 4 and 5 below for the first 2 modes.

where $\bar{f}, \mathrm{~S}_{\mathrm{f}}$, and $\mathrm{CI}_{0.95}$ are namely the mean frequency, its standard deviation, and the confidence interval, calculated according to a t-distribution.

In Tables 6 and 7, damping ratios for the two configurations are presented. As expected, the foamed tubes show higher damping ratios than the empty ones.

where $\bar{\xi}, \mathrm{S}_{\xi}$, and $\mathrm{CI}_{0.95}$ are namely the mean damping ratio, its standard deviation and the confidence interval, calculated according to a t-distribution.

ANOVA (analysis of variance) test is performed on the experimental results for each mode in order to estimate both the influence of filling (empty or foamed) and the diameter on the damping ratio mean value [10]. The results of the analysis were reported in the tables below.

According to the Tables 8 and 9 , the $P$-value is high for the diameter factor; therefore, the damping ratio mean values show no significant differences passing from one diameter to

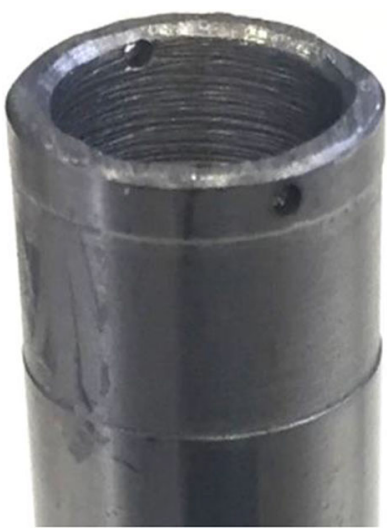

Fig. 4 Stepped tube at the connection interface with the flange 
Table 2 Preliminary test case

\begin{tabular}{lll}
\hline Preliminary test & Conf. A & Conf. B \\
\hline Number of samples & 3 & 3 \\
Foam & No & No \\
\hline
\end{tabular}

Table 3 Main test case

\begin{tabular}{lll}
\hline & Conf. A & Conf. B \\
\hline Number of samples & 3 & 3 \\
Foam & Yes & Yes \\
Foam length $(\mathrm{mm})$ & 180 & 180 \\
Foam material $(/)$ & Alulight & Alulight \\
\hline
\end{tabular}

Table 4 Eigenfrequencies configuration A

\begin{tabular}{lllll}
\hline D. 8 mm & Filling & $\bar{f}[\mathrm{~Hz}]$ & $\mathrm{S}_{\mathrm{f}}[\mathrm{Hz}]$ & $\mathrm{CI}_{0.95}[\mathrm{~Hz}]$ \\
\hline Mode 1 & Empty & 226.2 & 0.4 & {$[225.2 ; 227.2]$} \\
Mode 1 & Foamed & 218.7 & 1.3 & {$[215.4 ; 222.0]$} \\
Mode 2 & Empty & 1398.1 & 0.8 & {$[1396.0 ; 1400.1]$} \\
Mode 2 & Foamed & 1360.9 & 3.4 & {$[1352.6 ; 1369.3]$} \\
\hline
\end{tabular}

another one [11]. On the contrary, the $P$-value is nearly null for the filling factor, which can be considered, then, as the main source of variation of damping ratio among those analyzed.

This behavior is clearly visible in the interval plot (Figs. 5 and 6) for both the modes.

\subsection{FEM models}

Afterwards, the experimental tests were simulated by FEM models for each tube (see Fig. 7 below). The main aim of these FEM models was to properly reproduce the dynamic behavior of the tubes in order to have a good agreement between the experimental and the simulated FRFs.

Table 5 Eigenfrequencies configuration B

\begin{tabular}{lllll}
\hline D. $12 \mathrm{~mm}$ & Filling & $\bar{f}[\mathrm{~Hz}]$ & $\mathrm{S}_{\mathrm{f}}[\mathrm{Hz}]$ & $\mathrm{CI}_{0.95}[\mathrm{~Hz}]$ \\
\hline Mode 1 & Empty & 334.2 & 0.9 & {$[332.0 ; 336.3]$} \\
Mode 1 & Foamed & 321.3 & 0.8 & {$[319.3 ; 323.3]$} \\
Mode 2 & Empty & 2057.3 & 3.6 & {$[2048.3 ; 2066.3]$} \\
Mode 2 & Foamed & 2000.2 & 4.1 & {$[1990.1 ; 2010.3]$} \\
\hline
\end{tabular}

Table 6 Damping ratio configuration A

\begin{tabular}{lllll}
\hline D. 8 mm & Filling & $\bar{\xi}[/]$ & $\mathrm{S}_{\xi}[/]$ & $\mathrm{CI}_{0.95}[/]$ \\
\hline Mode 1 & Empty & 0.00089 & 0.00021 & {$[0.00038 ; 0.00141]$} \\
Mode 1 & Foamed & 0.00285 & 0.00007 & {$[0.00268 ; 0.00302]$} \\
Mode 2 & Empty & 0.00116 & 0.00011 & {$[0.00089 ; 0.00143]$} \\
Mode 2 & Foamed & 0.01292 & 0.00386 & {$[0.00332 ; 0.02251]$} \\
\hline
\end{tabular}

Table 7 Damping ratio configuration B

\begin{tabular}{lllll}
\hline D. $12 \mathrm{~mm}$ & Filling & $\bar{\xi}$ & $\mathrm{S}_{\xi}$ & $\mathrm{CI}_{0.95}[/]$ \\
\hline Mode 1 & Empty & 0.00035 & 0.00028 & {$[-0.00036 ; 0.00105]$} \\
Mode 1 & Foamed & 0.00220 & 0.00150 & {$[-0.00152 ; 0.00592]$} \\
Mode 2 & Empty & 0.00076 & 0.00013 & {$[0.00045 ; 0.00108]$} \\
Mode 2 & Foamed & 0.01713 & 0.00386 & {$[0.00754 ; 0.02672]$} \\
\hline
\end{tabular}

Table 8 Analysis of variance for damping ratio (mode 1)

\begin{tabular}{llllll}
\hline Source & DF & SS & MS & $\begin{array}{l}F- \\
\text { value }\end{array}$ & $\begin{array}{l}P \text { - } \\
\text { value }\end{array}$ \\
\hline Diameter [mm] & 1 & 0.000001 & 0.000001 & 2.04 & 0.187 \\
Filling & 1 & 0.000011 & 0.000011 & 20.67 & 0.001 \\
Error & 9 & 0.000005 & 0.000001 & & \\
Total & 11 & 0.000017 & & & \\
\hline
\end{tabular}

There are four parts modelled flange, tube, bolts, and foam (only for the filled tube case). The bolts are the only part that is not 3D; they are modelled as beam with an assigned section, and they are fixed to a circular partition of the flange (pink areas in Fig. 6), representing the real contact area (surface of the steel washers), by a coupling connection (Fig. 8).

The connection of the flange to the circular plate (see Fig. 2) is modelled by an "encastre" constraint on four circular partition, representing the real contact area (area of the steel washers) on the rear part of the flange (red areas in Fig. 9 below).

The welding between the tube and the flange is modelled by means of tie connections, applied on the effective welding areas (see highlighted surfaces in Fig. 10).

Table 9 Analysis of variance for damping ratio (mode 2)

\begin{tabular}{llllll}
\hline Source & DF & SS & MS & $\begin{array}{l}F- \\
\text { value }\end{array}$ & $\begin{array}{l}P \text { - } \\
\text { value }\end{array}$ \\
\hline Diameter [mm] & 1 & 0.000011 & 0.000011 & 1.30 & 0.284 \\
Filling & 1 & 0.000593 & 0.000593 & 70.55 & 0.000 \\
Error & 9 & 0.000076 & 0.000008 & & \\
Total & 11 & 0.000680 & & & \\
\hline
\end{tabular}


Fig. 5 Interval plot of damping ratio (mode 1)
Interval Plot of Damping Ratio - Mode 1

$95 \% \mathrm{Cl}$ for the Mean

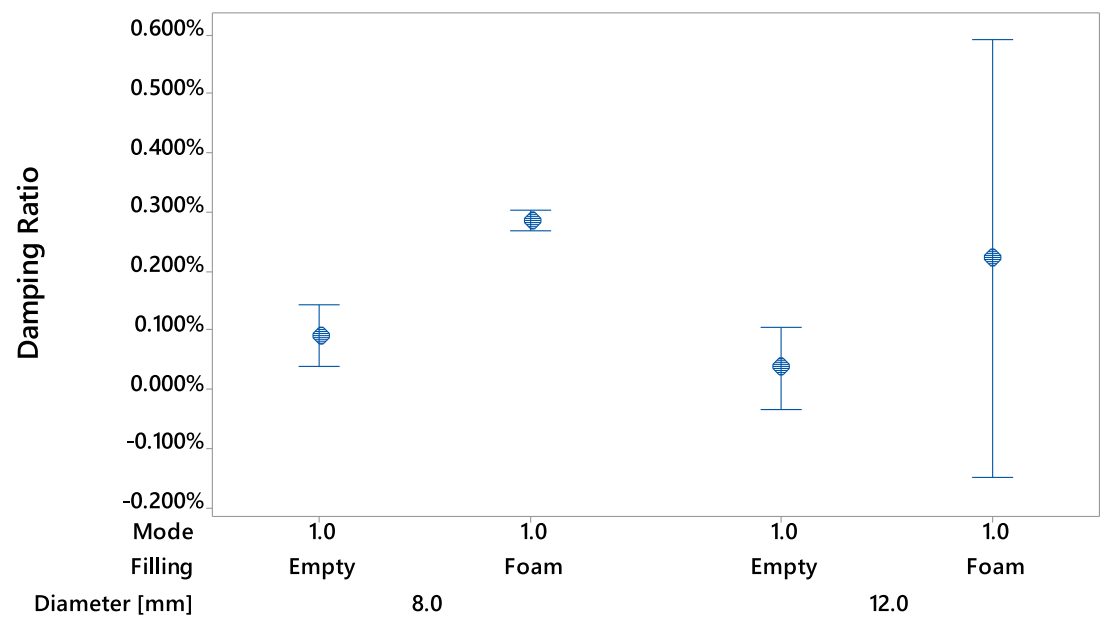

The connection between the inner surface of the tube and the metal foam is also modelled by a tie constraint, applied all over the outer surface of the foam. This kind of constraint does not allow the relative motion of the foam respect the tube, but in the real component, the foam is not bonded to the tube, so slip can occur. This relative motion could explain the high damping ratio for modes with a parabolic shape (e.g., mode shape 2 in Fig. 11), in which there is a high energy dissipation due to friction, as suggested by Baumeister et al. [8]. To model the relative motion would be computational expensive, so it is better to focus on the damping model and the material properties of the foam.

For all the parts, except the metal foam, a steel material is used (see Table 10 below).

As already said, it is strictly important to model properly the damping behavior of the foamed tubes. For this reason, a well-validated damping model as Rayleigh one has been used for the metal foam. This model allows to calculate the critical damping factor $h_{i}$ as a function of natural frequencies $\omega_{i}$ of the structure, but it requires user-defined input parameters $\alpha$ and $\beta$, which represent the mass proportional contribution and the stiffness proportional contribution, respectively:

$h_{i}=\frac{\alpha}{2 \omega_{i}}+\frac{\beta \omega_{i}}{2}$

For the above reasons, coefficients $\alpha$ and $\beta$ have been tuned in order to match experimental and simulated FRFs (see Fig. 11). Coefficients $\alpha$ and $\beta$, for both the configurations, are reported in Table 11.

The extracted mode shapes 1 and 2 are shown in Figs. 12 and 13 for both the configurations of foamed tubes.
Fig. 6 Interval plot of damping ratio (mode 2)
Interval Plot of Damping Ratio - mode 2 $95 \% \mathrm{Cl}$ for the Mean

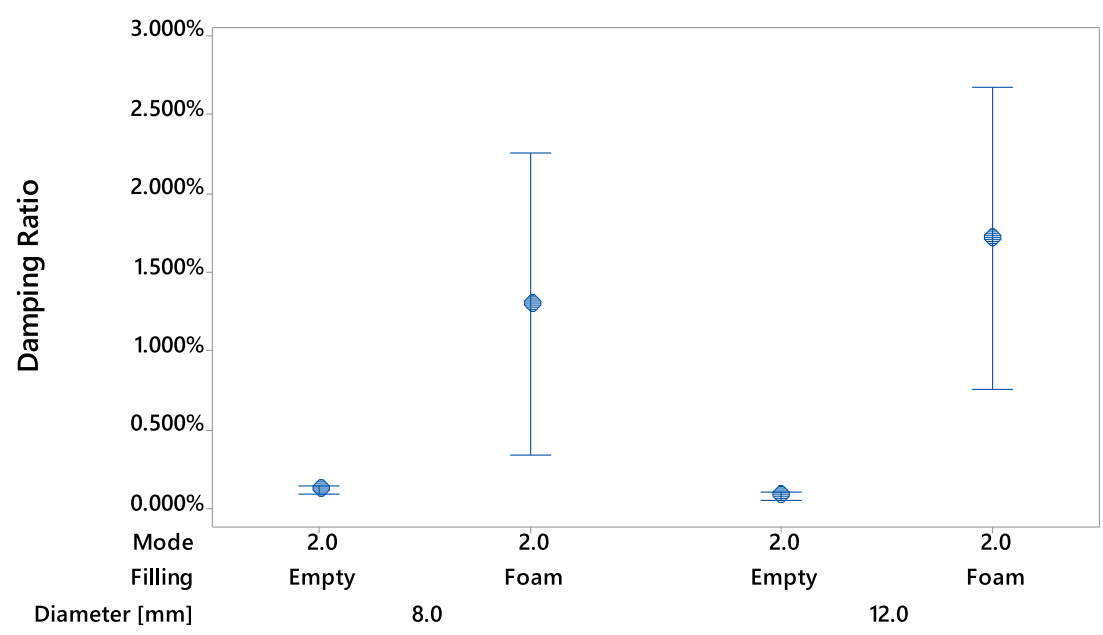




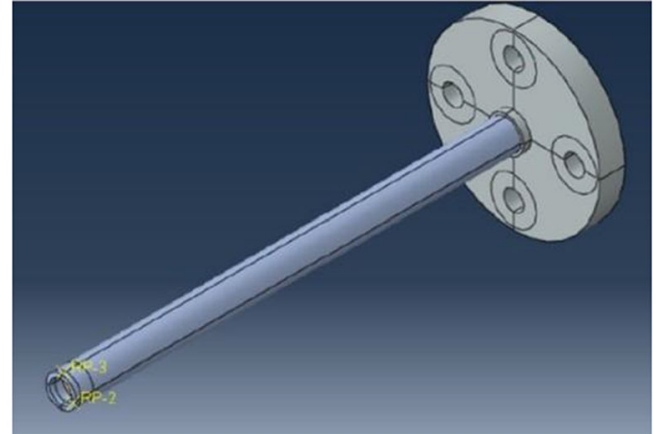

Fig. 7 FEM model

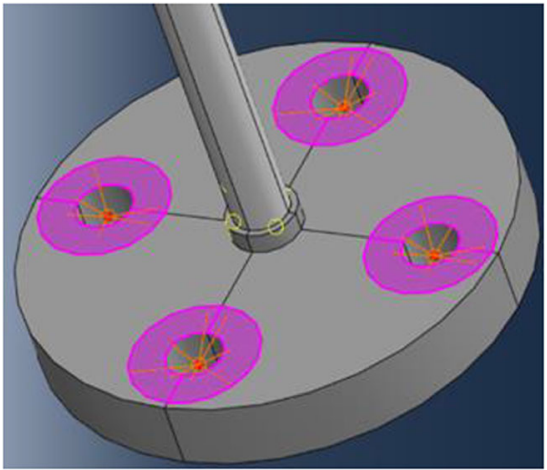

Fig. 8 Coupling connection

For sake of simplicity, the comparison between experimental FRF and simulated one is shown only for the conf. A of foamed tube (Fig. 14), but the same procedure is applied for conf. B and similar results were obtained.

\subsection{Damping predictive model}

The $\alpha$ and $\beta$ values, obtained in the previous phase, were used to develop a predictive model, which allows to estimate Rayleigh coefficients on foam filled structures made of one or more circular tubes. A linear relationship between Rayleigh coefficients and $\Delta f$ is proposed. $\Delta f$ is the difference between

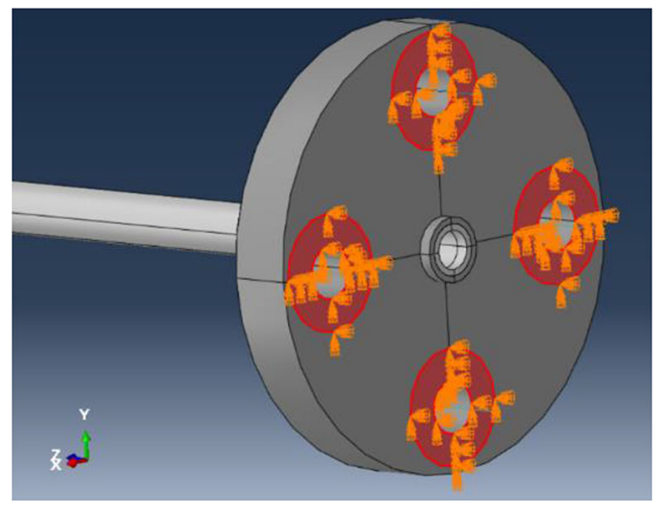

Fig. 9 Encastre constraint

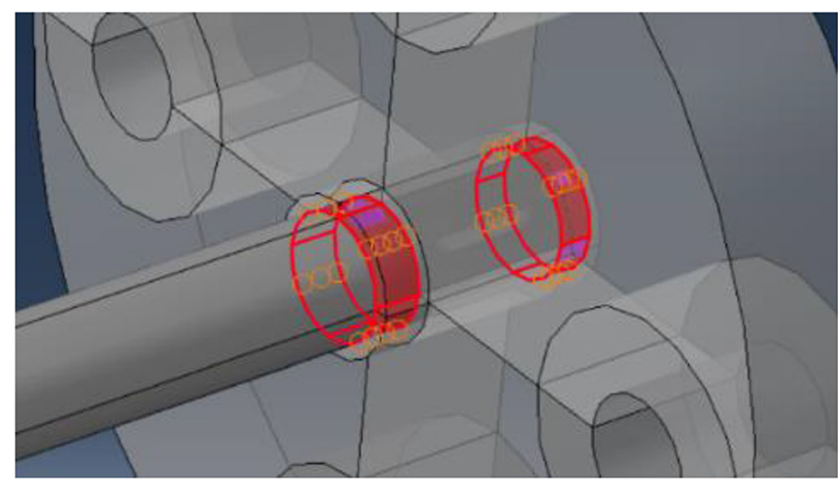

Fig. 10 Tie connection between tube and flange

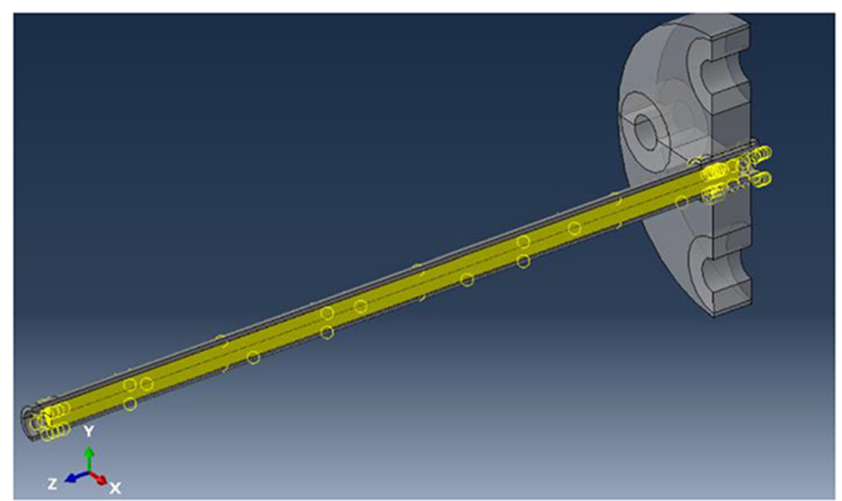

Fig. 11 Tie connection between tube and foam

the frequencies of a pair of vibrational modes of the structure. In order to have a correct estimation, the chosen pairs have to respect vibrational similitude. Indeed, the two mode shapes of conf. A should be similar to the two modes of conf. B (see Figs. 9 and 10). A linear formulation for $\alpha$ and $\beta$ was conceived, as shown in the following formulas:

$\alpha=m_{\alpha} \times \Delta f+q_{\alpha}$

$\beta=m_{\beta} \times \Delta f+q_{\beta}$

where $m_{\alpha}, m_{\beta}$ and $q_{\alpha}, q_{\beta}$ have been extracted during the previous experimental phase. It is important to point out that these four coefficients depend only on the materials of the tested circular tubes (see Table 12 below).

On the basis of the collected data for the tested tubes and of the previous equations, it is possible to build a 3D plot (Fig. 15),

Table 10 Material properties of FEM model

\begin{tabular}{lll}
\hline & Steel & Foam \\
\hline Young's modulus & $206 \mathrm{GPa}$ & $1.35 \mathrm{GPa}$ \\
Poisson's ratio & 0.33 & 0.35 \\
Density & $7600 \mathrm{~kg} / \mathrm{m}^{3}$ & $350 \mathrm{~kg} / \mathrm{m}^{3}$ \\
Structural damping & 0.001 & 0 \\
\hline
\end{tabular}


Table 11 Rayleigh coefficients

\begin{tabular}{lll}
\hline Foam & Conf. A & Conf. B \\
\hline$\alpha$ & 345 & 487 \\
$\beta$ & 0.00054 & 0.0008 \\
\hline
\end{tabular}

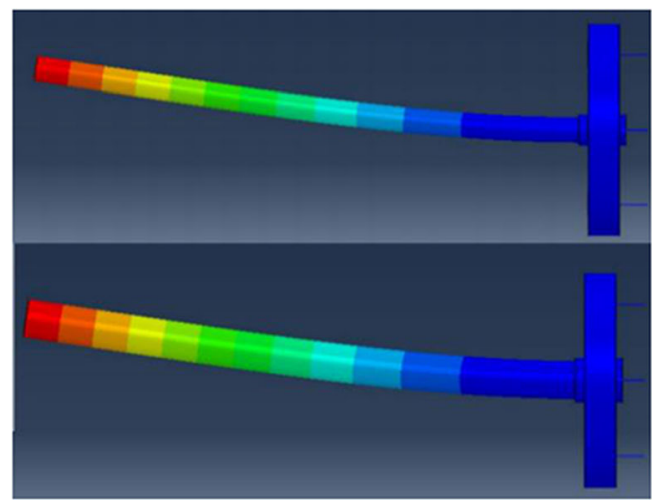

Fig. 12 Mode shape 1 for foamed tubes (conf. A and conf. B)

which represents the variability of the critical damping factor $h$ as a function of frequency $f$ and $\Delta f$.

The proposed predictive model allows to calculate Rayleigh damping coefficients to be used in the FEM model to simulate the vibrational behavior of aluminum foamed tubes. This is a big advantage because, if the same materials are used, no further experimental tests are needed for the design of new components. In addition, this model can be applied to more complex structures with respect to the tested ones, as it will be shown in the next paragraph.

\section{Results and discussion}

In this section, the validation of the proposed damping predictive model is presented. Two representative structures were considered, and the validation was performed

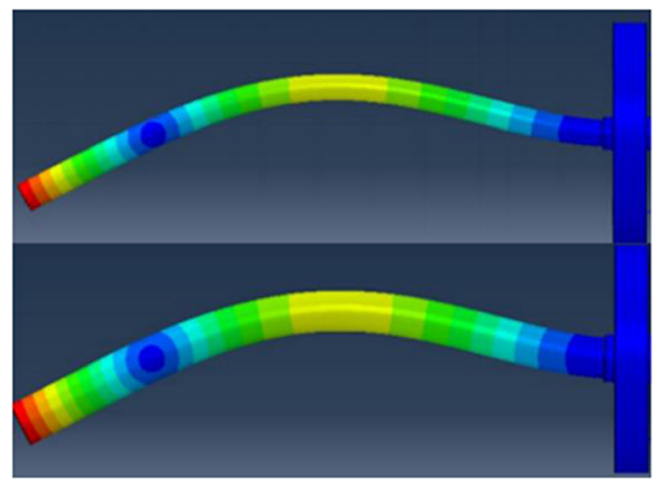

Fig. 13 Mode shape 2 for foamed tubes (conf. A and conf. B)

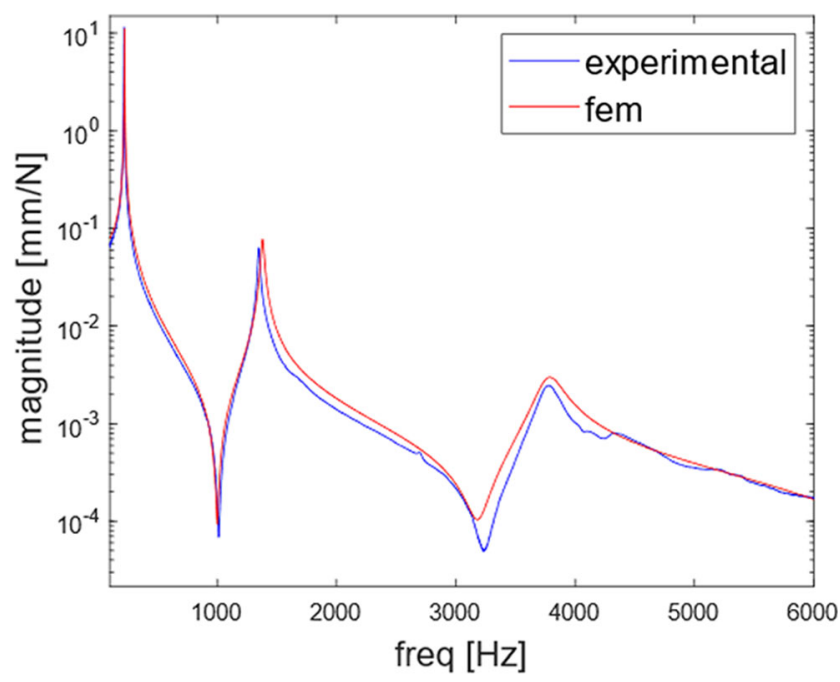

Fig. 14 FRF validation for foamed tube (conf. A)

Table 12 Coefficients for steel-Alulight material

on the basis of the comparison between the simulated and experimental FRFs.

\subsection{Model validation on foamed tube}

In order to have a first validation of the damping predictive model, further configuration for the foamed tubes was evaluated (see Table 13 below):

Firstly, the eigenfrequencies were extracted by a FEM model of the tube, so no damping input data were required. The natural frequencies of the first 2 modes are used as input parameters of the proposed $\Delta f$-based predictive model, in

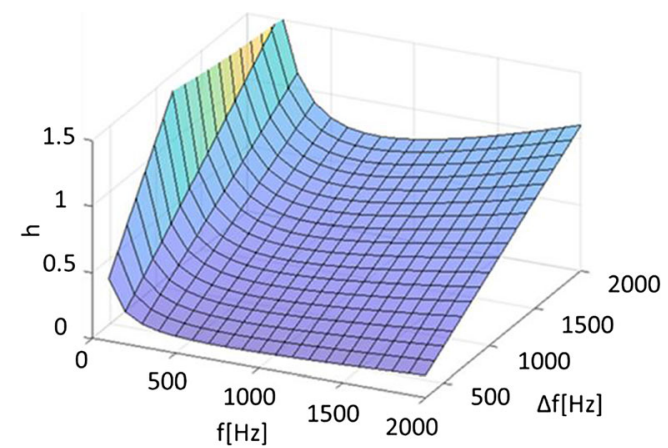

Fig. 15 Critical damping factor 3D plot 
Table 13 Configuration $\mathrm{C}$

\begin{tabular}{ll}
\hline Parameter & Conf. C \\
\hline Tube length (mm) & 190 \\
Tube outer diameter (mm) & 12 \\
Tube thickness (mm) & 2 \\
Tube material $(/)$ & Steel \\
Foam length (mm) & 180 \\
Foam material (/) & Alulight \\
\hline
\end{tabular}

order to estimate the Rayleigh damping coefficients $\alpha$ and $\beta$. This pair of modes was chosen as their shapes were similar to those ones used for the determination of the coefficients $\mathrm{m}_{\alpha \text {, }}$ $\mathrm{m}_{\beta}, \mathrm{q}_{\alpha}$, and $\mathrm{q}_{\beta}$.

Afterwards, the so-calculated Rayleigh coefficients were used in a FEM model to simulate the damping behavior of the $12 \times 2 \mathrm{~mm}$ foamed tube and to calculate its FRF. In order to validate the predictive model, the simulated FRF is compared with the experimental one. As shown in Fig. 16 below, there is a good agreement between the frequency response functions. In fact, the damping behavior, in terms of magnitude of the peaks, was properly estimated.

In addition, the percentage error $E_{m, i}$ on the estimation of the magnitude of the $i$ th mode is calculated as follows:

$E_{m, i}=\frac{m_{F E M, i}-m_{\exp , i}}{m_{\exp , i}} \times 100$

where $m_{\mathrm{FEM}, \mathrm{i}}$ and $\mathrm{m}_{\mathrm{exp}, \mathrm{i}}$ are the experimental and simulated magnitudes of the $i$ th mode, respectively. Results are reported in Table 14 below:

It can be seen in Table 11 that the peak amplitude of the $1 \mathrm{st}$ mode was predicted quite well; instead, the 2 nd mode showed a higher error, maybe related to the lower similarity of the chosen mode with the 2 nd mode of the predictive model.

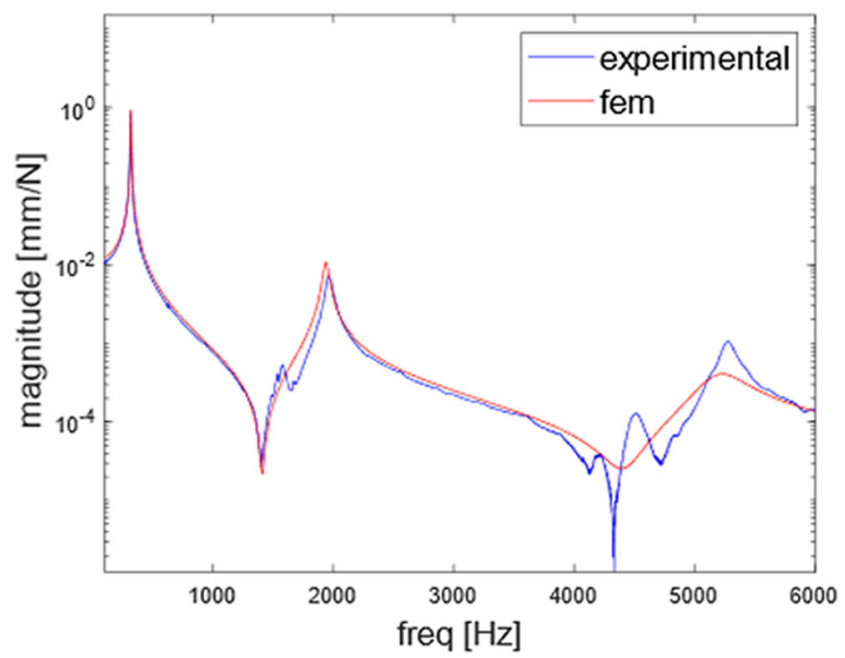

Fig. 16 FRF validation for foamed tube (conf. C)
Table 14 Magnitude error conf. C

\begin{tabular}{llll}
\hline Mode number & $\mathrm{m}_{\exp }[\mathrm{mm} / \mathrm{N}]$ & $\mathrm{m}_{\text {FEM }}[\mathrm{mm} / \mathrm{N}]$ & $\mathrm{E}_{\mathrm{m}}[/]$ \\
\hline 1 & 0.852243 & 0.933667 & $+10 \%$ \\
2 & 0.007164 & 0.010880 & $+52 \%$ \\
\hline
\end{tabular}

\subsection{Model validation on complex structure}

The previous procedure was even applied to a more complex structure, in order to verify if the damping prediction formulation still works properly. A structure, named D-cage, composed of aluminum foamed tubes (conf. B), was chosen for this phase (see Fig. 17).

Like the previous case, the eigenfrequencies and the mode shapes of the structure are calculated by FEM models. Comparing the mode shapes, it is possible to hypothesize a similarity between the first mode of the single tube and the first mode of the D-cage and, again, a similarity between the second mode of the single tube and the fourth mode of the Dcage (see Figs. 18 and 19).

Since the structure was quite different than previous ones, it was necessary to verify the similitude. In order to assess if the chosen mode shapes were similar to those ones used for the determination of the coefficients $\mathrm{m}_{\alpha}, \mathrm{m}_{\beta}, \mathrm{q}_{\alpha}$, and $\mathrm{q}_{\beta}$, the modal assurance criterion (MAC) analysis [7] was used. Indeed, the chosen pair of mode shapes of the foamed tube (conf. B) and of the D-cage was compared by means of the following formula:

$\operatorname{MAC}(r, q)=\frac{\left|\left\{\psi_{A}\right\}_{r}^{T}\left\{\psi_{X}\right\}_{q}^{*}\right|^{2}}{\left(\left\{\psi_{A}\right\}_{r}^{T}\left\{\psi_{A}\right\}_{r}^{*}\right)\left(\left\{\psi_{X}\right\}_{q}^{T}\left\{\psi_{X}\right\}_{q}^{*}\right)}$

In Eq. 5, MAC is calculated as the normalized scalar product of two set of eigenvectors $\left\{\psi_{A}\right\}$ and $\left\{\psi_{X}\right\}$, corresponding

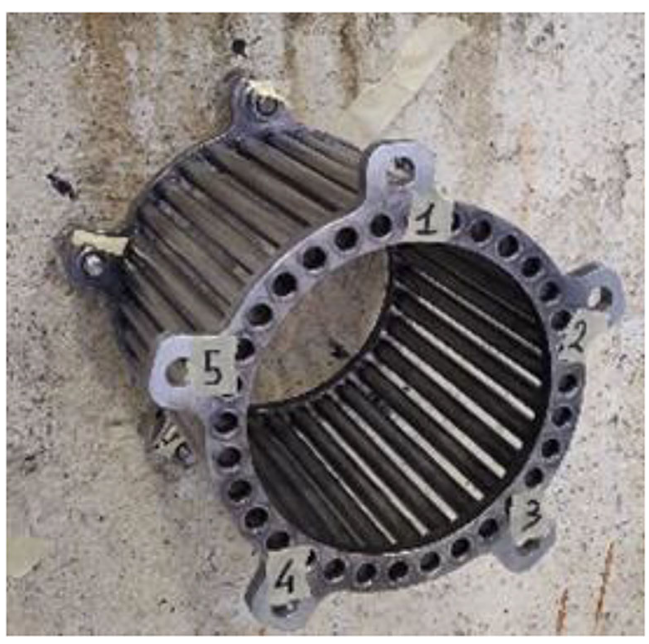

Fig. 17 D-cage structure 


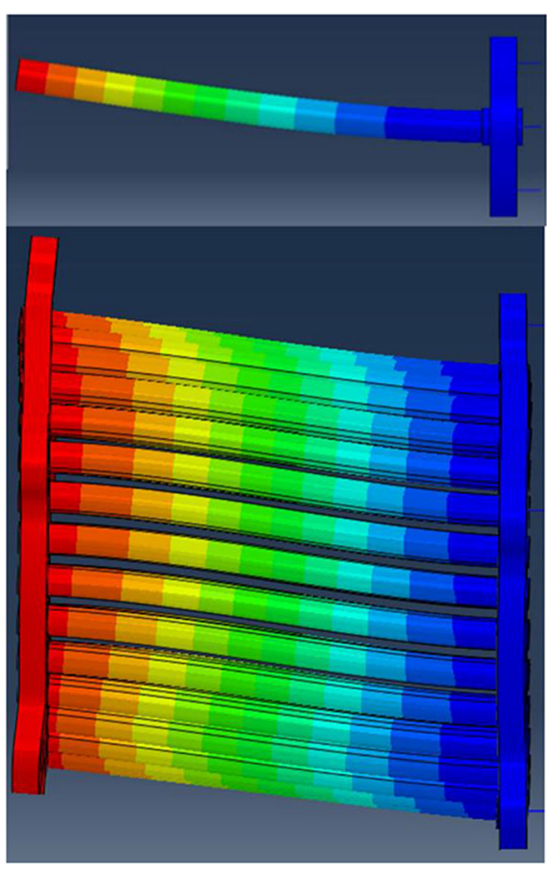

Fig. 18 Mode shape 1 for foamed tubes (conf. B) and D-cage

to the mode shapes to compare. The MAC takes value from 0 (representing no similarity) to 1 (representing perfect similarity) [9]. In Table 15 below, MAC results are reported.

Both MAC are high enough to consider the modes to be similar, so $\Delta f$ can be calculated as the difference of the first and the fourth eigenfrequency found for the D-cage (Table 16).

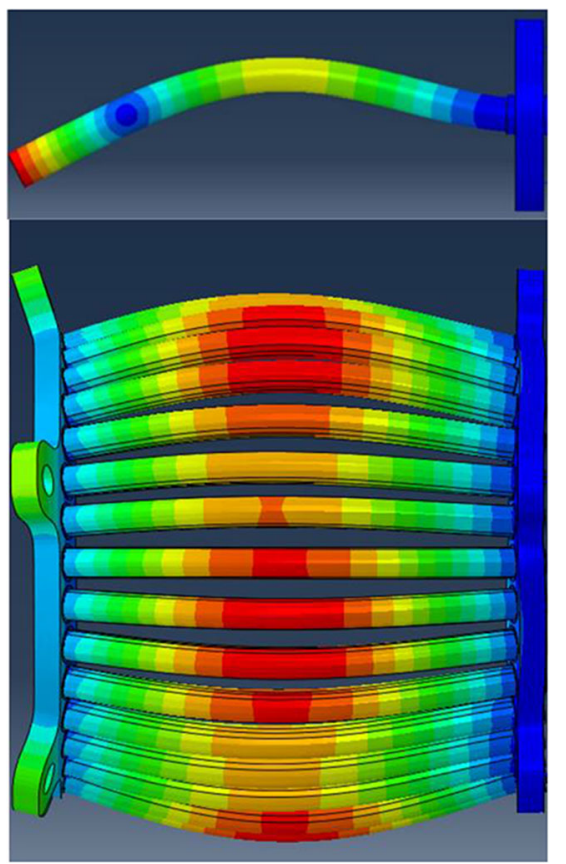

Fig. 19 Mode shape 2 for foamed tube (conf. B) and mode shape 4 for Dcage
Table 15 MAC results

\begin{tabular}{lll}
\hline Conf. & Mode number & Mode number \\
\hline B & 1 & 2 \\
D-cage & 1 & 4 \\
MAC & 0.9342 & 0.8350 \\
\hline
\end{tabular}

Table 16 Magnitude error D-cage

\begin{tabular}{llll}
\hline Mode number & $\mathrm{m}_{\exp }[\mathrm{mm} / \mathrm{N}]$ & $\mathrm{m}_{\mathrm{FEM}}[\mathrm{mm} / \mathrm{N}]$ & $\mathrm{E}_{\mathrm{m}}[/]$ \\
\hline 1 & 0.032784 & 0.030550 & $-7 \%$ \\
2 & 0.024606 & 0.024041 & $-2 \%$ \\
\hline
\end{tabular}

As for the foamed tubes, simulated FRF was extracted by FEM simulations and compared to the experimental one. The simulated FRF (Fig. 20), based on the damping coefficients extracted by the proposed damping formulation, fits quite well the experimental one (see Fig. 6); therefore, the proposed model for the identification of $\alpha$ and $\beta$ seems to work properly even for a more complex structure.

The good agreement of the FRFs is confirmed by the results in terms of percentage magnitude error $\mathrm{E}_{\mathrm{i}}$.

\section{Conclusion}

In the proposed research work, a model to predict damping properties of metal foams in simple and complex structures was presented. In the first part of the paper, experimental tests and FEM simulations were performed on simple structures, i.e., aluminum foam filled tubes, in order to define their dynamic properties, in

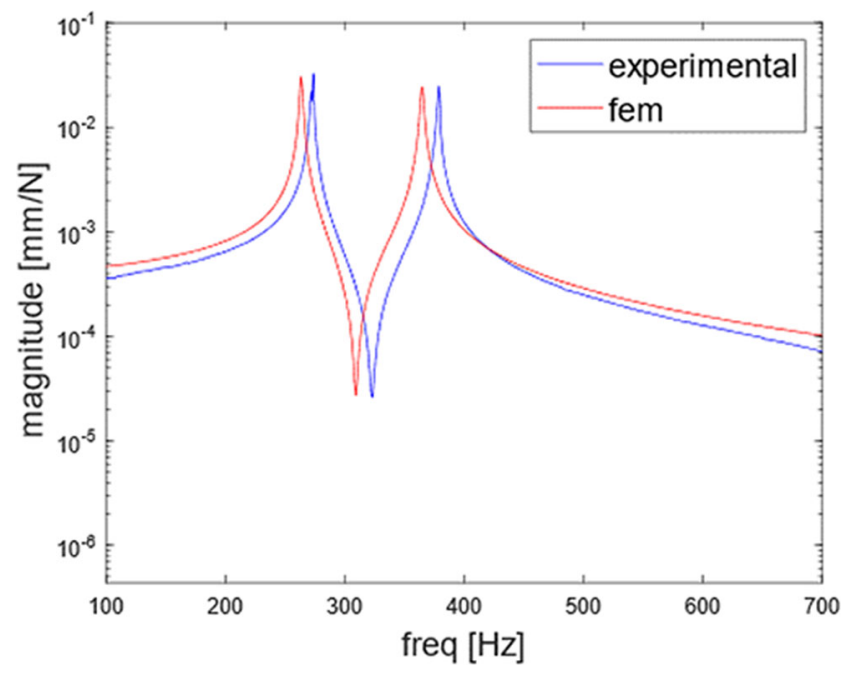

Fig. 20 FRF validation for D-cage structure 
terms of critical damping factor $h_{i}$. The foamed tubes were all made with the same materials, but different diameters and thicknesses were tested and their FRFs experimentally measured. The collected data were then used in FEM models, in order to replicate properly the tests and to identify Rayleigh damping coefficients $\alpha$ and $\beta$, which best fit the simulated FRFs with the experimental ones. The so-obtained Rayleigh coefficients were used in the damping predicting formulation, which allows to estimate $\alpha$ and $\beta$ (and therefore critical damping factor $h_{i}$, too) as a function of $\Delta f$, i.e., the difference between the frequencies of a pair of modes of a no-tested structure. The chosen mode shapes have to be consistent (have to respect similitude) with those ones used for the determination of coefficients of the conceived predictive model $m_{\alpha}, m_{\beta}, q_{\alpha}$, and $q_{\beta}$. The main goal of this novel formulation is to estimate critical damping factor $h_{i}$ on different or more complex structures with respect to the original ones, without the necessity of repeating experimental tests. In fact, it is just required a frequency FEM analysis on the new structure as long as the materials do not change, in order to calculate $\Delta f$ and obtain the related $h_{i}$ by the proposed formulation.

In the second part, the proposed model was validated, considering two different structures. The first one was an aluminum foam filled tube, but with different dimensions. On the contrary, the second evaluated structure was more complex since it was made of several foamed tubes. In both the cases, the formulation was exploited to predict coefficients $\alpha$ and $\beta$ to feed the FEM and for obtaining the simulated FRFs, without the necessity of experimental tests on the evaluated structure. Finally, the adequacy of the model was confirmed by the comparison of simulated FRFs and experimental ones.

Nomenclature $\alpha$, Mass proportional Rayleigh coefficient; $\beta$, Stiffness proportional Rayleigh coefficient; $\mathrm{CI}_{0.95}$, Confidence interval at $95 \%$; Conf., Configuration of tested tubes; $D$., Outer diameter of the tube; $\Delta f$, Difference between first two eigenfrequencies; $E_{m, i}$, Percentage magnitude error of the $i$ th mode; $\bar{f}$, Mean frequency; FEM, Finite element method; FRF, Frequency response function; $h$, Critical damping factor; $h_{i}$, Critical damping factor of $i$ th mode; $m_{\alpha}$, Gradient in $\alpha$ linear equation; $m_{\beta}$, Gradient in $\beta$ linear equation; $m_{\exp , i}$, Experimental magnitude of the $i$ th mode; $m_{\mathrm{FEM}, i}$, Simulated magnitude of the ith mode; $\bar{\xi}$, Mean damping ratio; $\left\{\psi_{\mathrm{A}}\right\}$, Eigenvector mode $\mathrm{A} ;\left\{\psi_{\mathrm{X}}\right\}$, Eigenvector mode $X ; q_{\alpha}$, Intercept in $\alpha$ linear equation; $q_{\beta}$, Intercept in $\beta$ linear equation; $S_{\xi}$, Standard deviation of damping ratio; $S_{f}$, Standard deviation of frequency; $\omega_{i}$, Natural frequency of $i$ th mode

Author contribution Paolo Albertelli conceived the research, made the analysis, and developed the model. Stefano Esposito wrote the paper and did the revision. Valerio Mussi arranged the experimental setup and carried out the experimental tests. Mattia Torta and Massimo Goletti carried out the experimental tests and made the preliminary analyses. Michele Monno carried out the proofreading.

Funding Open access funding provided by Politecnico di Milano within the CRUI-CARE Agreement. The research was funded by Capellini Srl company.

Data availability Data are not available since the project was developed in collaborations with a company.
Code availability No specific code was developed for release.

\section{Declarations}

Compliance with ethical standards This research follows ethical standards.

Consent to participate The authors agree.

Consent for publication The authors gave consent for publication.

Conflict of interest The authors declare no competing interests.

Open Access This article is licensed under a Creative Commons Attribution 4.0 International License, which permits use, sharing, adaptation, distribution and reproduction in any medium or format, as long as you give appropriate credit to the original author(s) and the source, provide a link to the Creative Commons licence, and indicate if changes were made. The images or other third party material in this article are included in the article's Creative Commons licence, unless indicated otherwise in a credit line to the material. If material is not included in the article's Creative Commons licence and your intended use is not permitted by statutory regulation or exceeds the permitted use, you will need to obtain permission directly from the copyright holder. To view a copy of this licence, visit http://creativecommons.org/licenses/by/4.0/.

\section{References}

1. Mevada H, Patel D (2016) Experimental determination of structural damping of different materials. Procedia Eng 144:110-115

2. Ashby MF (2016) Metal foams : a design guide. Butterworth Heinemann 3069:264

3. Goletti M, Mussi V, Rossi A, Monno M (2014) Procedures for damping properties determination in metal foams to improve FEM modeling. Procedia Mater Sci 4:233-238

4. Arora V (2014) Structural damping identification method using normal FRFs. Int J Solids Struct 51:133-143

5. Aggogeri F, Borboni A, Merlo A, Pellegrini N, Ricatto R (2017) Vibration damping analysis of lightweight structures in machine tools. Materials (Basel). 10

6. Strano M, Marra A, Mussi V, Goletti M, Bocher P (2015) Endurance of damping properties of foam-filled tubes. Materials (Basel) 8:4061-4079

7. Ewins DJ (2000) Modal testing: theory, practice and Application. 2nd ed., London, U.K

8. Baumeister J, Monno M, Goletti M, Mussi V, Weise J. Dynamic behavior of hybrid APM (advanced pore morphology foam) and aluminum foam filled structures. Metals, 2012

9. Pastor M, Binda M (2012) Harcarik T. Modal assurance criterion, MMaMS

10. Montgomery D (2001) Design and analysis of experiments. 5th ed. John Wiley and Sons

11. Labonnote N, Rønnquist A, Malo KA (2013) Experimental evaluations of material damping in timber beams of structural dimensions. Wood Sci Technol 47:1033-1050

Publisher's note Springer Nature remains neutral with regard to jurisdictional claims in published maps and institutional affiliations. 